\title{
Designing Floor-Layouts with the Assistance of Curious Agents
}

\author{
Ewa Grabska ${ }^{1}$, Katarzyna Grzesiak-Kopeć ${ }^{2}$, and Grażyna Ślusarczyk ${ }^{1}$ \\ 1 Jagiellonian University, Institute of Computer Science, Nawojki 11, \\ 30-072 Cracow, Poland \\ uigrabsk@cyf-kr.edu.pl, grazyna@ii.uj.edu.pl \\ 2 Jagiellonian University, The Faculty of Physics, Astronomy and Applied Computer \\ Science, Department for Information Technology, \\ Reymonta 4, 30-059 Cracow, Poland \\ katarzyna.grzesiak-kopec@uj.edu.pl
}

\begin{abstract}
The paper deals with visual computational design in which emergence is a key to creativity. The presented framework for conceptual design uses shape grammars and curious agent assistants. The intelligent agents perceive the changing environment and emergent phenomena that occur in it. Interacting with each other and the designer they look for the most original and plausible solutions to a given design task. The approach is illustrated by the example of a designing floor-layouts.
\end{abstract}

\section{Introduction}

This paper presents a new framework for visual kind of creative conceptual design with shape grammars and curious agent assistants. It takes advantage of visual shape grammar computations, emergent phenomena which enhance creativity and curiosity of intelligent agents searching for novel solutions. The approach deals with various external conditions while the design process takes place. The interaction with the environment can happen both through the autonomous designer's decisions and through the approved curious agents prompts. The framework is illustrated by the example of a designing floor-layouts.

\section{Dynamic Creative Design and Shape Grammars}

One of the most important phenomena which enhances creativeness is emergence. In design, these features which are not explicitly represented but emerge from a design structure are called emergent (Fig. 11). The ability to recognise properties which are not originally intended in design is an important aspect of human visual perception and plays a key role in the interaction between the designer and his/her drawing or sketch. In most computational systems emergence is limited to generation and observation of emergent features. Using shape grammars as generative systems allows us not only to generate and recognise emergent elements but fed them back into computation as well [4. The formal 


\section{A}

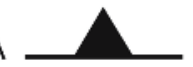

$\mathrm{B}$

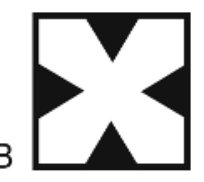

Fig. 1. (A) Single object. (B) Configuration of four copies of the given object which implies an emergent cross.

model [2] for dynamic design using shape grammars has been verified by a dedicated application DesignAnalizer2D 3. The classified emergent elements [2] are detected on-line by the system and new design rules containing them (emergent rules) are defined. We have programmed shape grammars with the use of a control diagram which specifies the order of applying grammar rules.

\section{Curious Design Agents Architecture}

In our approach agents are to be the designer's assistants in creative design. By an agent we understand a computer system situated in an environment and capable of autonomous actions in this environment in order to satisfy its design task 6. Its inside structure consists of processes connected with perception, evaluation, planning, and action which affects the environment. By creative designs we understand here designs which are in a way new (unexpected, original) and useful (valuable, valid) [1. In order to perform the task, each curious agent has to be intelligent: perceive its environment and respond to changes that occur in it, exhibit goal-directed behaviour and interact with other agents [7].

A curious agent observes its environment, gathers perceptual inputs and changes its internal state appropriately. On the one hand, the goal of our agent is to determine the novelty of a situation which means to validate the originality of the current environmental state with respect to the history. On the other hand, on the basis of the agent's interest in the present situation it has to identify unexpected consequences of actions. Thus, the agent's interest depends on the gained knowledge, while its curiosity can be understood as a motivation to do the further research. To support curiosity, the minimal agent architecture must include some form of memory. The mentioned requirements suggest using two different types of memory: a short-term memory to store recent experiences and a long-term one to store agent's generalised experiences from the past [5]. Both novelty and surprise of the situation can be computed by comparing the current state of an agent with its previous experiences. Information about the environment state and history, and the interest degree are taken into account in a decision making process. New goals of an agent are specified and the chosen goal is mapped into an action. In the proposed solution the short-term memory is implemented as a store of variables. The long-term memory has a form of a neural network called Self-Organising Map (SOM). It should be stressed that different implementations of a short-term and a long-term memory influence the learning ability of the agent and can lead to different curious behaviour. 


\section{Curious Agent Assistant}

We expect that the curious agent assistants extend our possibilities to obtain interesting and valid design solutions. Two curious agent assistants are presented to support the designer in searching for original floor-layout designs by promoting the most creative solutions to a given design task.

Our environment consists of a set of design rules with a control diagram specifying the order of their application and the artefact to be designed in a given phase of generation. The environment is inspected by two curious agent assistants: inquisitive ( $i$-agent) and sage (s-agent). The i-agent is responsible for introducing new emergent rules into a shape grammar and the s-agent is in charge of a generation of possible designs. In order to improve the learning process of the agents and to incorporate the designer knowledge and preference more efficiently, a list of the best solutions, proposed by agents, is presented to the designer and he/she chooses one of them. Thus, the agents future decisions depend not only on their mutual interactions but on the interaction with the designer as well.

Let us consider the example of designing a floor plan. The designer has to determine two initial requirements: an overall shape of a floor plan (Fig. 2A) and a number of rooms. Assume that the required number of rooms is equal to 5. Applying design rules shown in Fig. 2, in a sequence 1, 3, 2, 3 the designer obtains an initial floor-layout with 5 rooms (Fig. 2B).
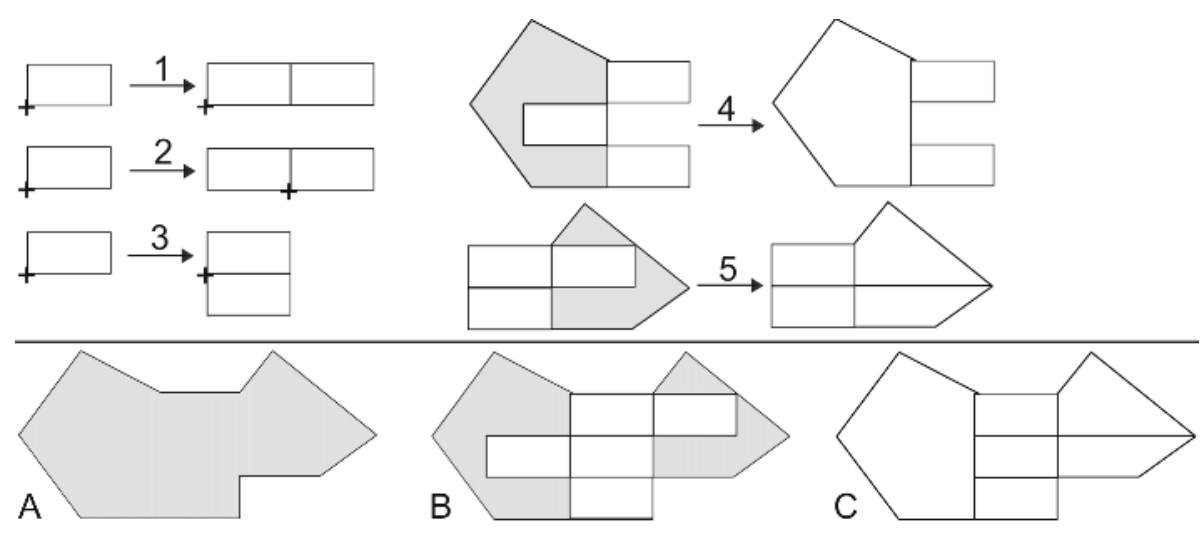

Fig. 2. (1-3) Regular grammar rules; (4,5) Emergent rules; (A) Given floor plan; (B) Early floor-layout with 5 rooms generated; (C) The designed floor-layout

The i-agent looks for new interesting design rules based on emergent elements which have come on the scene in the current step of generation. It localises emergent holes [2] (empty spaces), determines the design elements which constitute them and generates a set of possible rules to replace them. Then, basing on its experience and knowledge, it proposes the most appropriate design rules to the s-agent (Fig. 2 rule numbers 4 and 5). 
The s-agent evaluates the rules proposed by the i-agent by applying them to the current generation and verifying whether the obtained solution is still valid. For example, it checks whether the generated floor-layout does not include overlapping areas or the number of rooms is different from the required one. Knowing the current generation step, the s-agent takes into account also rules which are already in the grammar, and evaluates them in the same manner as emergent ones. Having compared both emergent and regular solutions it recommends the best one, which satisfies all conditions, to the designer as the next step in the present generation. It also points out the shapes in the generation to which the rule should be applied. Fig. $2 \mathrm{C}$ shows one of the possible final solutions after applying the following rules: $1,3,2,3,4,5$.

Now, let us consider alternative flows of events. Since not all new rules proposed by the i-agent are acceptable to the s-agent, there may be more than one iteration in the negotiations between two assistants. If all attempts at mediation fail, either the best from the obtained solutions or none of them is chosen.

\section{Conclusions}

In this paper we presented a new approach to conceptual design. In computational design the emphasis on the dynamic design context is crucial to artificial creativity. Visual computations are situated in a context indirectly through the designer working with the application or more explicitly through curious agents. The intelligent agents perceive the changing environment and emergent phenomena that occur in it. Interacting with each other and the designer they look for the most original and plausible solutions to a given design task.

\section{References}

1. Grabska, E., Ślusarczyk, G. and Grześ, P.: Dynamic design with the use of intelligent agents. Proceedings of the 4th International Conference on Computer Recognition Systems CORES'05. Springer (2005) 827-834

2. Grzesiak-Kopeć, K.: Emergent elements in periodic designs: An attempt at formalization. Design Computing and Cognition '04. Kluwer Academic Publishers (2004)

3. Grzesiak-Kopeć, K.: Visual designing of graphical models with the use of emergent elements. PhD thesis (in polish). Faculty of Electrical Engineering, Automatics, Computer Science and Electronics. AGH University of Science and Technology (2005)

4. Knight, T.: Interaction in visual design computing. Visual and Spatial Reasoning in Design III. The invited paper (2004)

5. Saunders, R.: Curious design agents and artificial creativity. PhD thesis. Faculty of Architecture. The University of Sydney (2002)

6. Wooldridge, M. J. and Jennings, N.R.: Intelligent agents: Theory and practice. The Knowledge Engineering Review. Vol. 10(2) (1995)

7. Wooldridge, M. J.: Intelligent agents. Multiagent systems: a modern approach to distributed artificial intelligence. MIT Press. Cambridge. MA (1999) 